\title{
COMPETITIVE FORMS OF SYMMETRY BREAKING IN LINEAR ANTIFERROMAGNETIC SYSTEMS
}

\author{
W.J. CASPERS \\ Center for Theoretical Physics, Twente University of Technology, Enschede, The Netherlands \\ and \\ W. MAGNUS \\ Instituut voor Theoretische Fysica, University of Leuven, B-3030 Leuven, Belgium
}

Received 24 September 1984

Two different forms of symmetry breaking are considered for linear antiferromagnetic systems $\left(S=\frac{1}{2}\right)$. Their relative stability is examined by considering small fluctuations in the harmonic oscillator approximation. Imaginary frequencies correspond with an unstable phase, and the ground state represents an absolute minimum of the total energy, including contributions from the zero-point fluctuations.

\section{Introduction}

Extensive study has been made of the so-called singlet-pair states (SPS) of linear antiferromagnetic spin $\frac{1}{2}$ systems with nearest and next-nearest neighbour interactions. There are also examples of other linear systems that demonstrate this type of ordering, but which have an interaction of longer range ${ }^{1-8}$ ). The SPS does not possess a classical analogue.

Intuitive approaches of quantum mechanical ordering in magnetic systems start with a given classical ordering (e.g. the Néel state). Fluctuations around this ordered state, considered as coupled quantized harmonic oscillators, lead to the well-known spin-wave model (SWM) of (anti)ferromagnetism ${ }^{9}$ ). The SPS may be considered as the zeroth-order approximation of another approach in which the pairs are treated as oscillators. For those Hamiltonians for which the SPS are exact ground states these oscillators are uncoupled, but for other interactions there exists a coupling. In the harmonic oscillator approximation for this coupling we have an alternative of the SWM.

Which of the two methods leads to the best approximation of the ground state may be decided on the basis of the characteristics of the corresponding frequency spectra. In this work we consider the existence of imaginary frequencies as an indication of instability of the phase. If both phases only have 
positive frequencies the lowest total energy, including the zero-point fluctuations, should give, in principle, the (stable) ground state, the other phase being metastable in this case. There may be corrections to this recipe in the case that the results for one phase show obvious anomalies. In the border-line case for which there exists one frequency $\omega=0$, in a real spectrum, there exists a soft mode. The method using singlet-pairs may be readily generalized by considering groups of $4,6, \ldots, 2 n$ spins.

The existence of two competitive forms of antiferromagnetic ordering seems to be a characteristic of linear spin $\frac{1}{2}$ systems. These forms have a different type of symmetry breaking: whereas in the "classical" ordering there exists a non-vanishing sublattice magnetization, the SPS, or its generalizations, does not show this effect, i.e. the expectation value of the spin (magnetization) for all lattice sites equals zero. So in the "classical" ordering the rotational symmetry in spinor space is always broken, in combination with a broken translational symmetry, whereas in the other phase (SPS-like) only the translational symmetry has vanished ${ }^{10}$ ).

On the basis of Goldstone's theorem we may then conclude that the spectrum of elementary excitations in the "classical" case does not show a gap, whereas for the ordering of the SPS type there may be a non-vanishing distance to the first excited state ${ }^{11}$ ). Recent calculations for the Majumdar-Ghosh model give strong support for this idea ${ }^{8}$ ).

In this paper we make a systematic comparison between the two methods for linear antiferromagnetic systems with nearest and next-nearest neighbour interactions, which are, in the general case, chosen to be anisotropic. The anisotropy for the nearest and next-nearest neighbour interactions is chosen to be the same, the overall ratio of the two couplings being a variable parameter of the model.

In section 2 we give a general description of the Hamiltonian of the system. The zeroth-order states and the corresponding harmonic oscillator approximation for the two approaches constitute the subject of section 3 . The material of that section is the basis of the criterion for stability outlined in section 4. It turns out that in the case that both spectra are real one of the approaches results in a soft mode. The soft mode suggests the possibility of spontaneous deformation, which is always in accordance with the symmetry of the stable phase.

In a final section we give a discussion of our analysis in relation with results of the literature.

\section{General Hamiltonian}

We consider a general type of interaction between nearest and next-nearest 
neighbour pairs, with the restriction that both interactions are proportional. This restriction may not be an essential one but gives some simplifications in the calculations and provides us with a subset of Hamiltonians for which the ground state is exactly known: the singlet-pair state.

For an even number of spins: $2 \mathrm{~N}$, our Hamiltonian reads

$$
\begin{aligned}
H= & 4 \sum_{i=1}^{2 N}\left\{\left[(1+\gamma) S_{i x} S_{i+1, x}+(1-\gamma) S_{i y} S_{i+1, y}\right]+(1+\Delta) S_{i z} S_{i+1, z}\right\} \\
& +4 \delta \sum_{i=1}^{2 N}\left\{\left[(1+y) S_{i x} S_{i+2, x}+(1-\gamma) S_{i y} S_{i+2, y}\right]+(1+\Delta) S_{i z} S_{i+2, z}\right\} \quad\left(S_{i}=\frac{1}{2}\right),
\end{aligned}
$$

which may also be conveniently written:

$$
\begin{aligned}
H= & 2 \sum_{i=1}^{2 N}\left\{\left[\gamma\left(S_{i+} S_{i+1,+}+S_{i-} S_{i+1,-}\right)+\left(S_{i+} S_{i+1,-}+S_{i-} S_{i+1,+}\right)\right]\right. \\
& \left.+2(1+\Delta) S_{i z} S_{i+1, z}\right\}+2 \delta \sum_{i=1}^{2 N}\left\{\left[\gamma\left(S_{i+} S_{i+2,+}+S_{i-} S_{i+2,-}\right)\right.\right. \\
& \left.\left.+\left(S_{i+} S_{i+2,-}+S_{i-} S_{i+2,+}\right)\right]+2(1+\Delta) S_{i z} S_{i+2, z}\right\}
\end{aligned}
$$

If one subdivides the system into groups of two neighbouring spins the total Hamiltonian may be written as a sum of two parts, one $\left(H_{0}\right)$ corresponding to the internal interactions within the cells and the other $\left(H^{\prime}\right)$ to the interactions between cells. Doing so one may write ${ }^{12}$ )

$$
\begin{aligned}
H_{0}=\sum_{k=1}^{N} & H_{0, k}, \quad H^{\prime}=\sum_{k=1}^{N} H_{k, k+1}^{\prime}, \\
H_{0, k}=2 & \gamma\left(S_{2 k-1,}, S_{2 k,+}+S_{2 k-1,-} S_{2 k,-}\right)+\left(S_{2 k-1,+} S_{2 k,-}+S_{2 k-1,-} S_{2 k,+}\right) \\
+ & \left.2(1+\Delta) S_{2 k-1, z} S_{2 k, z}\right] \\
H_{k, k+1}^{\prime}= & 2\left[\gamma\left(S_{2 k,+} S_{2 k+1,+}+S_{2 k,-} S_{2 k+1,-}\right)+\left(S_{2 k,+} S_{2 k+1,-}+S_{2 k,-} S_{2 k+1,+}\right)\right. \\
& \left.+2(1+\Delta) S_{2 k, z} S_{2 k+1, z}\right]+2 \delta\left[\gamma\left(S_{2 k-1,+} S_{2 k+1,+}+S_{2 k-1,-} S_{2 k+1,-}\right)\right. \\
& +\left(S_{2 k-1,+} S_{2 k+1,-}+S_{2 k-1,-} S_{2 k+1,+}\right)+2(1+\Delta) S_{2 k-1, z} S_{2 k+1, z} \\
& +\gamma\left(S_{2 k,+} S_{2 k+2,+}+S_{2 k,-} S_{2 k+2,-}\right)+\left(S_{2 k,+} S_{2 k+2,-}+S_{2 k,-} S_{2 k+2,+}\right) \\
& \left.+2(1+\Delta) S_{2 k, z} S_{2 k+2, z}\right] .
\end{aligned}
$$

On the basis of the expressions for the Hamiltonian in (2) and (3), the 
unperturbed state will be given in the next section. For the method based on the SPS, this state corresponds with pairs, the energy of which is determined by diagonalizing the $H_{0, k}$. This lowest state should be a singlet, which condition results in some restrictions on the values of $\gamma$ and $\Delta$. The coupling between the separate cells, treated like harmonic oscillators, will be given by $H^{\prime}$. In the alternative method (SWM) the strongest of the three interactions, either in the $x, y$ or $z$ direction determines the Néel state. Fluctuations around this Néel state, treated as coupled oscillators, result in another approach of the antiferromagnetic ground state ${ }^{9}$ ). Which of the two approaches is the best one is determined by conditions of stability already indicated in the introduction.

\section{Zeroth-order states and harmonic oscillator approximations}

As was already outlined in section 2 the system will be treated as a set of coupled harmonic oscillators. This will be done in two ways according to the definition of the free oscillators. In subsection 3.1 cells of 2 or 4 spins will constitute, in zero order, a set of uncoupled systems, the motion of which being described by a harmonic oscillator approximation. The number of oscillators per cell equals the number of excited states. Coupling between the cells will have the form, which is also an approximation, of a bilinear expression in terms of the boson creation and annihilation operators ${ }^{13}$ ) of the corresponding cells. In subsection 3.2 the standard SWM will be given, generalized for a system with an anisotropic interaction between nearest and next-nearest neighbours.

\subsection{Harmonic oscillator approximation with cells}

As a consequence of translational symmetry the secular problem for each cell is the same, so, for convenience in the notation, the problem will be solved for cell 1 , with spins 1 and 2 , in the case of cells of two spins. The relevant part of $H_{0}$, i.e. $H_{0,1}$, reads

$$
H_{0,1}=2\left\{\left[\gamma\left(S_{1+} S_{2+}+S_{1-} S_{2-}\right)+\left(S_{1+} S_{2-}+S_{1-} S_{2+}\right)\right]+2(1+\Delta) S_{1 z} S_{2 z}\right\}
$$

The solution of the corresponding secular problem is elementary and the eigenstates and eigenvalues are listed in table I. Use is made of a notation in which \pm denote eigenvalues $\pm \frac{1}{2}$ of the spin components $S_{1(2) z}$. In kets like $|+,-\rangle$ the first symbol refers to spin 1 and the second to spin 2 . Eigenstates of $H_{01}$ are denoted by $|n\rangle, n=0,1,2,3$, the corresponding energy eigenvalues by $\epsilon_{n}$.

Only the case for which $|0\rangle$ is the ground state will be considered, so the 
TABLE I

Eigenstates and eigenvalues of $H_{0.1}$

$\begin{array}{ll}|0\rangle=\frac{1}{\sqrt{ } 2}[|+,-\rangle-|-,+\rangle] & \epsilon_{0}=-(3+\Delta) \\ |1\rangle=\frac{1}{\sqrt{ } 2}[|+,-\rangle+|-,+\rangle] & \epsilon_{1}=1-\Delta \\ |2\rangle=\frac{1}{\sqrt{ } 2}[|+,+\rangle-|-,-\rangle] & \epsilon_{2}=1+\Delta-2 \gamma \\ |3\rangle=\frac{1}{\sqrt{ } 2}[|+,+\rangle+|-,-\rangle] & \epsilon_{3}=1+\Delta+2 \gamma\end{array}$

parameters $\gamma$ and $\Delta$ should obey

$$
\Delta>-2+|\gamma| \text {. }
$$

The energy distance to the first excited state, either $|2\rangle$ or $|3\rangle$, is given by: $2 \Delta+4-2|\gamma|$. For $\Delta=-2+|\gamma|$ there is no "gap" in the energy spectrum of one single cell and the harmonic oscillator approximation may be unreliable in this case.

Now an approximate representation of $H$ will be given in terms of boson operators $^{13}$ ), defined by the zeroth-order Hamiltonian $H_{0}=\sum_{k=1}^{N} H_{0, k}$, which will be written:

$$
\begin{aligned}
& H_{0}=-(3+\Delta) N+\sum_{k=1}^{N}\left[4 c_{1}^{+}(k) c_{1}(k)+2(2+\Delta-\gamma) c_{2}^{+}(k) c_{2}(k)\right. \\
& \left.\quad+2(2+\Delta+\gamma) c_{3}^{+}(k) c_{3}(k)\right], \\
& {\left[c_{j}\left(k_{1}\right), c_{l}^{+}\left(k_{2}\right)\right]=\delta_{j l} \delta_{k_{1} k_{2}},} \\
& {\left[c_{j}^{+}\left(k_{1}\right), c_{l}^{+}\left(k_{2}\right)\right]=\left[c_{j}\left(k_{1}\right), c_{l}\left(k_{2}\right)\right]=0, \quad j, l=1,2,3 .}
\end{aligned}
$$

A crucial element in the argument is the supposition that the total probability of finding a cell in one of its three excited states is small as compared to 1 , and that this condition is fulfilled consistently in the harmonic oscillator approximation.

For this case one can make a transformation of the spin operators $S_{1}, S_{2}$ into the boson operators by considering the effect of the spin operators on the states of table I. Elementary calculations give the results of table II.

In the harmonic oscillator approximation transitions between excited states should be neglected and consequently, on the basis of table II, one may construct the transformations of table III. 
Table II

Effect of spin operators on eigenstates of $H_{0,1}$.

\begin{tabular}{lll}
\hline$S_{1 z}|0\rangle=\frac{1}{2}|1\rangle$ & $S_{1+}|0\rangle=\frac{1}{2}(-|2\rangle-|3\rangle)$ & $S_{1-}|0\rangle=\frac{1}{2}(-|2\rangle+|3\rangle)$ \\
$S_{2 z}|0\rangle=-\frac{1}{2}|1\rangle$ & $S_{2+}|0\rangle=\frac{1}{2}(|2\rangle+|3\rangle)$ & $S_{2-}|0\rangle=\frac{1}{2}(|2\rangle-|3\rangle)$ \\
$S_{1 z}|1\rangle=\frac{1}{2}|0\rangle$ & $S_{1+}|1\rangle=\frac{1}{2}(|2\rangle+|3\rangle)$ & $S_{1-}|1\rangle=\frac{1}{2}(-|2\rangle+|3\rangle)$ \\
$S_{2 z}|1\rangle=-\frac{1}{2}|0\rangle$ & $S_{2+}|1\rangle=\frac{1}{2}(|2\rangle+|3\rangle)$ & $S_{2-}|1\rangle-\frac{1}{2}(-|2\rangle+|3\rangle)$ \\
$S_{1 z}|2\rangle=\frac{1}{2}|3\rangle$ & $S_{1+}|2\rangle=\frac{1}{2}(-|0\rangle-|1\rangle)$ & $S_{1-}|2\rangle=\frac{1}{2}(-|0\rangle+|1\rangle)$ \\
$S_{2 z}|2\rangle=\frac{1}{2}|3\rangle$ & $S_{2+}|2\rangle=\frac{1}{2}(|0\rangle-|1\rangle)$ & $S_{2-}|2\rangle=\frac{1}{2}(|0\rangle+|1\rangle)$ \\
$S_{1 z}|3\rangle=\frac{1}{2}|2\rangle$ & $S_{1+}|3\rangle=\frac{1}{2}(|0\rangle+|1\rangle)$ & $S_{1-\cdots}|3\rangle=\frac{1}{2}(-|0\rangle+|1\rangle)$ \\
$S_{2 z}|3\rangle=\frac{1}{2}|2\rangle$ & $S_{2+}|3\rangle=\frac{1}{2}(-|0\rangle+|1\rangle)$ & $S_{2-}|3\rangle=\frac{1}{2}(|0\rangle+|1\rangle)$ \\
\hline
\end{tabular}

TABLE III

Transformations of spin variables into boson operators.

\begin{tabular}{|c|c|}
\hline \multicolumn{2}{|c|}{$\begin{array}{c}S_{1 z}=\frac{1}{2}\left[c_{1}^{+}(1)+c_{1}(1)\right] \\
S_{2 z}=-\frac{1}{2}\left[c_{1}^{+}(1)+c_{1}(1)\right]\end{array}$} \\
\hline$-\frac{1}{2}\left[c_{2}^{+}(1)+c_{3}^{+}(1)+c_{2}(1)-c_{3}(1)\right]$ & $S_{1-}=-\frac{1}{2}\left[c_{2}^{+}(1)-c_{3}^{+}(1)+c_{2}(1)+c_{3}(1)\right]$ \\
\hline
\end{tabular}

Now the transformation of the interaction $H^{\prime}$ into boson variables is also possible. After a lengthy but straightforward calculation one finds

$$
\begin{aligned}
H^{\prime} & =\sum_{k=1}^{N} H_{k, k+1}^{\prime}=(1-2 \delta) \sum_{k=1}^{N}\left\{-(1-\Delta)\left[c_{1}^{+}(k) c_{1}(k+1)+c_{1}(k) c_{1}^{+}(k+1)\right.\right. \\
& \left.+c_{1}^{+}(k) c_{1}^{+}(k+1)+c_{1}(k) c_{1}(k+1)\right]-(1+\gamma)\left[c_{2}^{+}(k) c_{2}(k+1)+c_{2}(k) c_{2}^{+}(k+1)\right. \\
& \left.+c_{2}^{+}(k) c_{2}^{+}(k+1)+c_{2}(k) c_{2}(k+1)\right]-(1-\gamma)\left[c_{3}^{+}(k) c_{3}(k+1)+c_{3}(k) c_{3}^{+}(k+1)\right. \\
& \left.\left.-c_{3}^{+}(k) c_{3}^{+}(k+1)-c_{3}(k) c_{3}(k+1)\right]\right\} .
\end{aligned}
$$

The easiest way to diagonalize the total Hamiltonian $H=H_{0}+H^{\prime}$, in the approximation given by (6) and (7), is to make a transformation to wave-like excitations:

$$
\begin{aligned}
& c_{s}^{+}(k)=\frac{1}{\sqrt{ } N} \sum_{q} \mathrm{e}^{-\mathrm{i} k q} c_{s}^{+}(q), \quad c_{s}^{+}(q)=\frac{1}{\sqrt{ } N} \sum_{k} \mathrm{e}^{\mathrm{i} k q} c_{s}^{+}(k), \\
& c_{s}(k)=\frac{1}{\sqrt{ } N} \sum_{q} \mathrm{e}^{\mathrm{i} k q} c_{s}(q), \quad c_{s}(q)=\frac{1}{\sqrt{ } N} \sum_{k} \mathrm{e}^{-\mathrm{i} k q} c_{s}(k), \\
& q=\frac{2 \pi r}{N}, \quad r=-\left(\frac{N}{2}-1\right),-\left(\frac{N}{2}-2\right), \ldots,-1,0,1, \ldots, \frac{N}{2}, \quad N \text { even }, \\
& r=-\frac{N-1}{2},-\frac{N-3}{2}, \ldots,-1,0,1, \ldots, \frac{N-1}{2}, \quad N \text { odd. }
\end{aligned}
$$


In terms of the $c_{s}^{(+)}(q)$ the Hamiltonian reads

$$
\begin{aligned}
H= & -(3+\Delta) N+\sum_{q}\left\{2[2-(1-2 \delta)(1+\Delta) \cos q] c_{1}^{+}(q) c_{1}(q)\right. \\
& +2[(2+\Delta-\gamma)-(1-2 \delta)(1+\gamma) \cos q] c_{2}^{+}(q) c_{2}(q) \\
& +2[(2+\Delta+\gamma)-(1-2 \delta)(1-\gamma) \cos q] c_{3}^{+}(q) c_{3}(q) \\
& -(1-2 \delta)(1+\Delta)\left[\mathrm{e}^{\mathrm{i} q} c_{1}^{+}(q) c_{1}^{+}(-q)+\mathrm{e}^{-\mathrm{i} q} c_{1}(q) c_{1}(-q)\right] \\
& -(1-2 \delta)(1+\gamma)\left[\mathrm{e}^{\mathrm{i} q} c_{2}^{+}(q) c_{2}^{+}(-q)+\mathrm{e}^{-\mathrm{i} q} c_{2}(q) c_{2}(-q)\right] \\
& \left.+(1-2 \delta)(1-\gamma)\left[\mathrm{e}^{\mathrm{i} q} c_{3}^{+}(q) c_{3}^{+}(-q)+\mathrm{e}^{-\mathrm{i} q} c_{3}(q) c_{3}(-q)\right]\right\} .
\end{aligned}
$$

The elementary excitations corresponding with this approximated Hamiltonian will be created by proper linear combinations of $c_{s}^{+}(q)$ and $c_{s}(-q)$, and should obey:

$$
\left[H, x_{s}(q) c_{s}^{+}(q)+y_{s}(q) c_{s}(-q)\right]=\omega_{s}(q)\left[x_{s}(q) c_{s}^{+}(q)+y_{s}(q) c_{s}(-q)\right] \quad(\hbar=1) .
$$

Making use of the commutation relations for the $c_{s}^{(+)}(q)$, which are easily derived from (6) and (8):

$$
\begin{aligned}
& {\left[c_{s_{1}}^{+}\left(q_{1}\right), c_{s_{2}}\left(q_{2}\right)\right]=\delta_{s_{1} s_{2}} \delta_{q_{1} q_{2}},} \\
& {\left[c_{s_{1}}^{+}\left(q_{1}\right), c_{s_{2}}^{+}\left(q_{2}\right)\right]=\left[c_{s_{1}}\left(q_{1}\right), c_{s_{2}}\left(q_{2}\right)\right]=0,}
\end{aligned}
$$

one finds the following secular equations for $x_{s}(q), y_{s}(q)$ and $\omega_{s}(q), s=1,2,3$ :

$$
\begin{aligned}
& {\left[4-A_{1}(q)-\omega_{1}(q)\right] x_{1}(q)+A_{1}(q) y_{1}(q)=0, \quad A_{1}(q)=2(1-2 \delta)(1+\Delta) \cos q,} \\
& -A_{1}(q) x_{1}(q)-\left[4-A_{1}(q)+\omega_{1}(q)\right] y_{1}(q)=0, \\
& {\left[2(2+\Delta-\gamma)-A_{2}(q)-\omega_{2}(q)\right] x_{2}(q)+A_{2}(q) y_{2}(q)=0,} \\
& A_{2}(q)=2(1-2 \delta)(1+\gamma) \cos q, \\
& -A_{2}(q) x_{2}(q)-\left[2(2+\Delta-\gamma)-A_{2}(q)+\omega_{2}(q)\right] y_{2}(q)=0, \\
& {\left[2(2+\Delta+\gamma)-A_{3}(q)-\omega_{3}(q)\right] x_{3}(q)-A_{3}(q) y_{3}(q)=0,} \\
& A_{3}(q)=2(1-2 \delta)(1-\gamma) \cos q, \\
& +A_{3}(q) x_{3}(q)-\left[2(2+\Delta+\gamma)-A_{3}(q)+\omega_{3}(q)\right] y_{3}(q)=0,
\end{aligned}
$$

which give the following frequencies: 


$$
\begin{aligned}
& \omega_{1}^{2}(q)=16-8 A_{1}(q)=16[1-(1-2 \delta)(1+\Delta) \cos q], \\
& \omega_{2}^{2}(q)=4(2+\Delta-\gamma)[2+\Delta-\gamma-2(1-2 \delta)(1+\gamma) \cos q], \\
& \omega_{3}^{2}(q)=4(2+\Delta+\gamma)[2+\Delta+\gamma-2(1-2 \delta)(1-\gamma) \cos q]
\end{aligned}
$$

Before considering in detail the conditions under which the three branches given in (13) correspond with real frequencies, we first make some simplifications by restricting the range of the parameters $y$ and $\Delta$.

First of all, without loss of generality, we may always choose $\gamma \geqslant 0$ because the transformation $\gamma \rightarrow-\gamma$ is a simple rotation of all spin vectors around the $z$-axis over an angle $\pi / 2$. Now the condition (5), which should be fulfilled in the case of a singlet ground state for a pair, reads

$$
1+\Delta>-(1-\gamma)
$$

This implies that the coupling constants for $y$ and $z$ components in (1) cannot both be negative, so that in all cases considered two coupling constants for a neighbour pair are positive, and, apart from a trivial factor, could always chosen to be $1+\gamma$ and $1-\gamma$, and consequently $0 \leqslant \gamma \leqslant 1$. Summarizing we have the following conditions:

$$
0 \leqslant \gamma \leqslant 1, \quad \Delta>-2+\gamma
$$

Now the conditions under which all frequencies (13) are real read

$$
|1-2 \delta| \leqslant \frac{1}{|1+\Delta|}, \quad|1-2 \delta| \leqslant \frac{2+\Delta-\gamma}{2(1+\gamma)}
$$

Under these conditions the transformations to the boson variables defined by (10) may be performed. The coefficients $x_{s}(q)$ and $y_{s}(q)$ obey the relations

$$
x_{s}(q)=x_{s}(-q), \quad y_{s}(q)=y_{s}(-q), \quad x_{s}^{2}(q)-y_{s}^{2}(q)=1, \quad x_{s}(q) \text { and } y_{s}(q) \text { real }
$$

and the transformation leads to the following ground-state energy:

$$
E_{0}=-(3+\Delta) N-\sum_{s q} \omega_{s}(q) y_{s}^{2}(q)
$$

The value of the $\omega_{s}$ is given in (13), whereas the $y_{s}$ may be derived from (12). So one finally arrives at 


$$
E_{0}=-3(3+\Delta) N+\frac{1}{2} \sum_{s q} \omega_{s}(q) .
$$

For the isotropic case $(\gamma=\Delta=0)$ this reduces to

$$
E_{0}(\gamma=\Delta=0, \delta)=N\left[-9+\frac{3}{\pi} \int_{-\pi}^{+\pi} \mathrm{d} q \sqrt{1-(1-2 \delta) \cos q}\right],
$$

which formula gives the exact result: $-3 N$ for $\delta=\frac{1}{2}$.

The result (18) may be written in terms of the elliptic function of the second kind:

$$
\begin{aligned}
& F_{0}(\gamma=\Delta=0, \delta)=2 N\left[-\frac{9}{2}+\frac{6 \sqrt{2 \delta}}{\pi} E\left(\frac{2 \delta-1}{\delta}\right)\right], \quad \delta>\frac{1}{2}, \\
& E_{0}(\gamma=\Delta=0, \delta)=E_{0}(\gamma=\Delta=0,1-\delta), \quad 0 \leqslant \delta \leqslant \frac{1}{2} .
\end{aligned}
$$

A strictly analogous calculation may be performed for cells of 4 spins. We have only considered the isotropic case, i.e. $\gamma=\Delta=0$ (cf. formulas (1) and (2)). The corresponding Hamiltonian for the interactions within a representative cell of 4 spins takes the form

$$
H_{0,1}=4\left[S_{1} \cdot S_{2}+S_{2} \cdot S_{3}+S_{3} \cdot S_{4}+\delta\left(S_{1} \cdot S_{3}+S_{2} \cdot S_{4}\right)\right],
$$

in which the second index of $H_{0,1}$ denotes the first group of 4 spins: $\{1,2,3,4\}$.

Because of rotational invariance in spinor space, the total spin $S$ is a good quantum number. We restrict ourselves to the case $\delta>0$, which implies that the lowest eigenstate of $H_{0,1}$ is always a singlet $(S=0)$. The complete set of eigenstates of $H_{0,1}$ includes two singlets, 3 triplets $(S=1)$ and one quintet $(S=2)$ and consequently the cell has a total number of 15 excited states, which may be created by 15 different boson operators acting on the ground state.

In the linearized model only the three triplets play a role, apart from the singlet ground state, which has an energy

$$
\epsilon_{0}=-3-2 \delta-2 \sqrt{4 \delta^{2}-6 \delta+3} .
$$

The excitation energies for the three triplets are respectively given by

$$
\begin{aligned}
& \eta_{1}=\epsilon_{1}-\epsilon_{0}=2+2 \delta+2 \sqrt{4 \delta^{2}-6 \delta+3}-2 \sqrt{1+(1-\delta)^{2}}, \\
& \eta_{2}=\epsilon_{2}-\epsilon_{0}=2+2 \sqrt{4 \delta^{2}-6 \delta+3} \\
& \eta_{3}=\epsilon_{3}-\epsilon_{0}=2+2 \delta+2 \sqrt{4 \delta^{2}-6 \delta+3}+2 \sqrt{1+(1-\delta)^{2}} .
\end{aligned}
$$


Again one may introduce boson operators for these cells of four spins and formulate a zero-order Hamiltonian $H_{0}$ for the uncoupled cells in terms of these operators, the energy $\epsilon_{0}$ and the three excitation energies being given in (21). This leads to an expression analogous to (6). Then again the coupling between the cells may be expressed in terms of the boson operators. Introducing wave-like excitations and performing a diagonalization for modes with the same $q$-value one finally arrives at the value for the ground-state energy per spin in this harmonic oscillator approximation. The very lengthy calculations are omitted here. We only give in table IV of section 4 the numerical results: $\epsilon_{0}^{(4)}$ together with those for the case of cells of two spins $\epsilon_{0}^{(2)}$, also for isotropic interactions. The values for $\delta$ for the case of cells of two spins are restricted to the interval $[0,1]$ (cf. (15), $\gamma=\Delta=0$ ) and for 4 spins to the interval $[0,1.4]$, both conditions resulting in real frequencies for all modes. In the same table one finds the corresponding results in the spin-wave approximation.

\subsection{The spin-wave model}

The spin-wave model (SWM) gives a standard method to determine ground states and elementary excitations in the theory of (anti)ferromagnetism ${ }^{9,10}$ ). It is extensively discussed in the literature, but in the textbooks the interactions are mostly restricted to nearest neighbours. Here we give an outline of the generalization for the interactions given in (1).

The $z$-axis will be chosen as the quantization axis, which defines the zero-order state in the SWM. Stability conditions (i.e. all magnon modes should correspond with real frequencies) will put restrictions on the values of $\gamma, \Delta$ and $\delta$. These restrictions will be formulated at the end of this subsection.

First we make a simple unitary transformation that transforms the zero-order state into a ferromagnetic state:

$$
\begin{gathered}
U H U^{+}=\bar{H}, \quad U=\prod_{j=1}^{N} \sigma_{2 j, x}, \quad \sigma_{i x}=2 S_{i x}, \\
\bar{H}=4 \sum_{i=1}^{2 N}\left[(1+\gamma)\left(S_{i x} S_{i+1, x}+\delta S_{i x} S_{i+2, x}\right)\right. \\
\quad+(1-\gamma)\left(-S_{i y} S_{i+1, y}+\delta S_{i y} S_{i+2, y}\right) \\
\left.\quad+(1+\Delta)\left(-S_{i z} S_{i+1, z}+\delta S_{i z} S_{i+2, z}\right)\right]
\end{gathered}
$$

The spin-wave operators are defined according to approximate second quantization ${ }^{14}$ ): 


$$
\begin{aligned}
& \left.S_{j+}=\frac{1}{\sqrt{ } 2 N} \sum_{q} \mathrm{e}^{\mathrm{i} q j} c_{q}\right\} \quad 1 \leqslant j \leqslant 2 N, \\
& \left.S_{j-}=\frac{1}{\sqrt{ } 2 N} \sum_{q} \mathrm{e}^{-\mathrm{i} i j} c_{q}^{+}\right\} \quad q=\frac{\pi}{N} m, \quad m=-N+1,-N+2, \ldots, N-1, N \text {, } \\
& {\left[c_{q_{1}}, c_{q_{2}}\right]=\left[c_{q_{1}}^{+}, c_{q_{2}}^{+}\right]=0, \quad\left[c_{q_{1}}, c_{q_{2}}^{+}\right]=\delta_{q_{1} q_{2}} .}
\end{aligned}
$$

In the SWM only terms bilinear in the $c_{q}\left(c_{q}^{+}\right)$are retained, after substituting (23) into (22), which leads to a representation of $H$ of the form

$$
\begin{aligned}
\bar{H}= & -2 N(1-\delta)(1+\Delta)+\sum_{q}\left\{4[(1+\Delta)(1-\delta)+\gamma \cos q+\delta \cos 2 q] c_{q}^{+} c_{q}\right. \\
& \left.+2\left(\mathrm{e}^{\mathrm{i} q}+\gamma \delta \mathrm{e}^{2 \mathrm{i} q}\right) c_{q}^{+} c_{-q}^{+}+2\left(\mathrm{e}^{-\mathrm{i} q}+\gamma \delta \mathrm{e}^{-2 \mathrm{i} q}\right) c_{-q} c_{q}\right\}
\end{aligned}
$$

This expression for the total energy may be reduced by means of a Bogolyubov transformation to a sum of independent boson modes:

$$
b_{q}^{+}=F_{q} c_{q}^{+}+G_{q} c_{-q}, \quad\left|F_{q}\right|^{2}-\left|G_{q}\right|^{2}=1,
$$

which obey the same commutation rules as the $c_{q}^{(+)}$.

The solution of the secular problem

$$
\left[\bar{H}, b_{q}^{+}\right]=\omega_{q} b_{q}^{+}
$$

leads in a straightforward way to the following expression for the energy of the elementary excitations:

$$
\omega_{q}=4\left\{[(1+\Delta)(1-\delta)+\gamma \cos q+\delta \cos 2 q]^{2}-[\cos q+\gamma \delta \cos 2 q]^{2}\right\}^{1 / 2},
$$

and the value for the ground-state energy

$$
\begin{aligned}
\bar{E}_{0}= & -6 N(1-\delta)(1+\Delta)+\frac{1}{2} \sum_{q} \omega_{q} \\
= & 2 N\left[-3(1-\delta)(1+\Delta)+\frac{2}{\pi} \int_{0}^{\pi} \mathrm{d} q\left\{[(1+\Delta)(1-\delta)+\gamma \cos q+\delta \cos 2 q]^{2}\right.\right. \\
& \left.\left.-[\cos q+\gamma \delta \cos 2 q]^{2}\right\}^{1 / 2}\right] .
\end{aligned}
$$

For $\gamma=\Delta=0$ this reduces to 


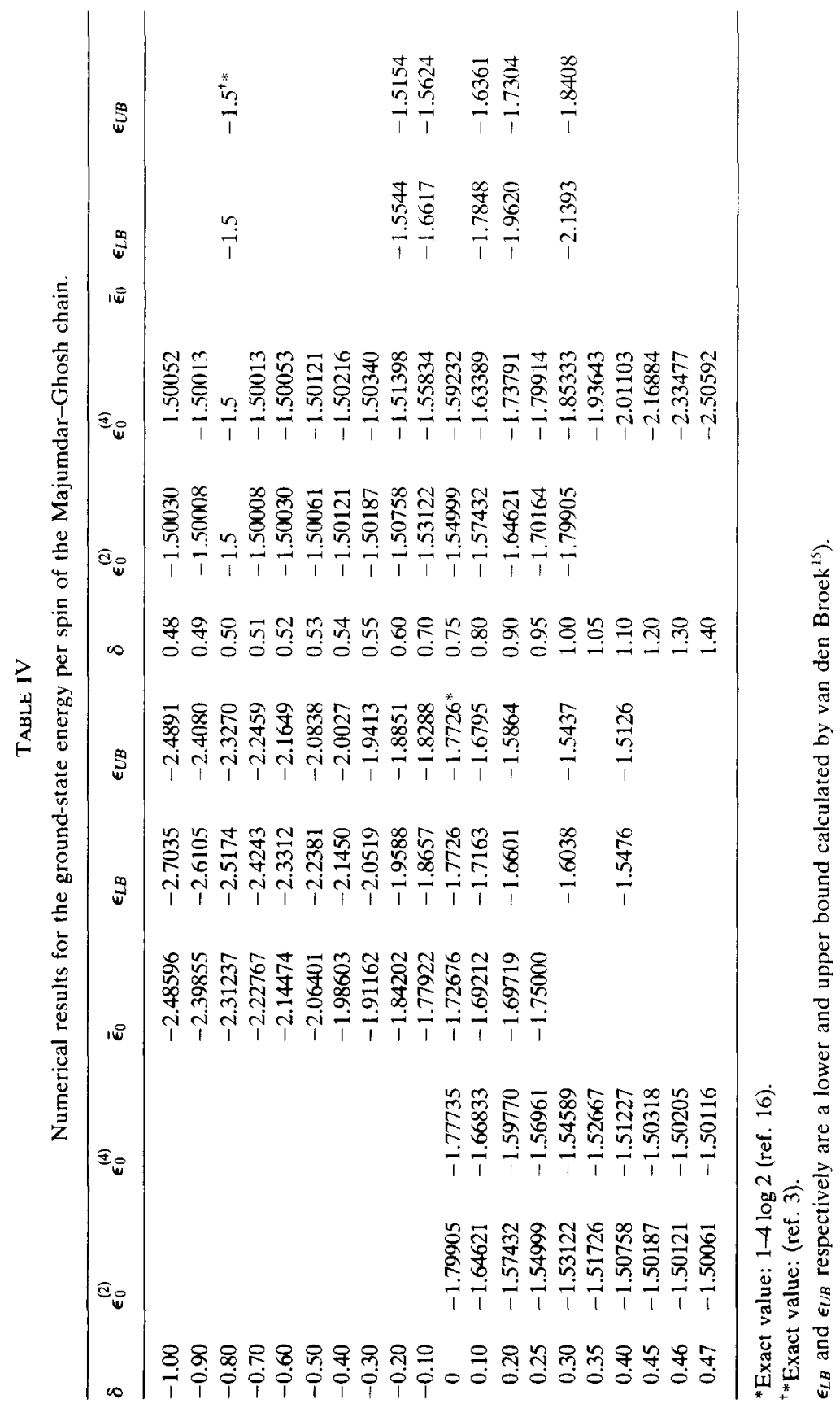




$$
\bar{E}_{0}=2 N\left\{-3(1-\delta)+\frac{1}{\pi}\left[\frac{|1-2 \delta|^{2}}{|\delta|} \arcsin \frac{2|\delta|}{|1-2 \delta|}+2 \sqrt{1-4 \delta}\right]\right\}
$$

This expression for the total energy of the ground state should be compared with the corresponding expressions in the harmonic oscillator approximation for cells, given in (17) and (18).

Before making a comparison between the two methods conditions for the stability of the modes in the SWM have to be formulated. These conditions being fulfilled, all frequencies $\omega_{q}$ in (27) are real, and they read

$$
\begin{aligned}
& 1+\Delta \geqslant 1+\gamma \text { or } 1+\Delta \leqslant-(1+\gamma) \frac{1+\delta}{1-\delta} \text { if }|\delta| \leqslant \frac{1}{4}, \\
& (1+\Delta)(1-\delta) \geqslant(1+\gamma) \frac{1+8 \delta^{2}}{8 \delta} \text { or }(1+\Delta)(1-\delta) \leqslant-(1+\gamma)(1+\delta) \\
& \text { if } \delta \geqslant \frac{1}{4} \\
& 1+\Delta \geqslant 1+\gamma \text { or } 1+\Delta \leqslant(1+\gamma) \frac{1+8 \delta^{2}}{(1-\delta) 8 \delta} \text { if } \delta \leqslant-\frac{1}{4} .
\end{aligned}
$$

The next section contains numerical results for the two types of approximation.

\section{Criterion for stability of the antiferromagnetic state}

Most attention will be paid, in this section, to the Majumdar-Ghosh chain, i.e. a chain with isotropic nearest and next-nearest neighbour interactions and an arbitrary ratio of the corresponding coupling constants. The Hamiltonian for this system is given by (1), with $\gamma=\Delta=0$. For cells of two spins we now may use (18) in the interval $0 \leqslant \delta \leqslant 1$, as was stated before, whereas the results for cells of 4 spins are restricted to: $0 \leqslant \delta \leqslant 1.4$. Spin-wave results hold for $\delta \leqslant \frac{1}{4}$, as follows from (29). The numerical results are listed in table IV. The results per spin for the harmonic oscillator approximation with cells of two spins are denoted by $\epsilon_{0}^{(2)}$, for cells of 4 spins by $\epsilon_{0}^{(4)}$ and in the spin-wave approximation by: $\bar{\epsilon}_{0}$.

The lowest value for the ground-state energy per spin for $\delta \geqslant 0$ is given by $\epsilon_{0}^{(4)}$. In the interval $0.10 \leqslant \delta \leqslant 0.25$, however, $\bar{\epsilon}_{0}$ seems to give a better approximation, but we should have serious doubts about these values because $\bar{\epsilon}_{0}(\delta)$ is not a monotonous function in this interval, as it should be according to ref. 15, from which paper it also follows that there is only one maximum, 
corresponding with $\delta=\frac{1}{2}$ and having the value $\epsilon\left(\frac{1}{2}\right)=-1.5$. Summarizing one may state that for $0 \leqslant \delta \leqslant 1.4$ the best approximation is given by $\epsilon_{0}^{(4)}$.

The SWM has soft modes for $q=0, \pi$ and for $\delta \geqslant 0$ this may give rise to spontaneous deformations, in accordance with the even and odd combinations of the approximate ground states constructed with fluctuating cells of two spins. Calculations with cells of 4 or more spins suggest a higher degeneracy of the ground state but this is not in accordance with the exact result for $\delta=\frac{1}{2}$, so we believe that this higher degeneracy only exists in this approximation and will finally disappear if all interactions between the harmonic oscillator modes are properiy taken care of. One may also remark that the exact result for $\delta=0$ is also well approximated by $\epsilon_{0}^{(4)}$. For $\delta=0$ the simple approximation with cells of two spins results in a soft mode for $q=0$, also well in accordance with the Néel state which is the basis for the SWM. For $\delta=1$ the soft mode corresponds with $q=\pi$, which suggests a doubling of the cell size. We know that the harmonic oscillator approximation for cells of 4 spins is correct for $\delta=1$, which confirms this result for the smaller cells. Furthermore for $\delta>1$ we do not know the classical ground state on which the SWM should be based. A structure of the type: $++--++--\ldots$ gives stable modes in the SWM approximation, only for $\delta \rightarrow \infty$.

The results of subsection 3.1 suggest that there will be an interval on the $\delta$-axis, with $\delta=\frac{1}{2}$ as an interior point, the length of the interval being a function of $\Delta$ and $\gamma$ (cf. (15)), for which the method with cells gives a better approximation to the ground-state energy as the SWM. This, because for $\delta=\frac{1}{2}$ we have an exact solution. In a sufficiently small neighbourhood of this point the representation of the symmetry group by the ground state will be the same, so that onc may expect that the symmetry breaking of the singlet-pair state will be a more general phenomenon, in such a sense that it is not only realized for a Majumdar-Ghosh chain, but also for other interactions.

This symmetry breaking always results in a gap of the spectrum of elementary excitations, in contradistinction to an ordering in which there exists a non-vanishing sublattice magnctization, for which there cannot exist a gap, according to Goldstone's theorem ${ }^{10,11}$ ). Preliminary studies of two- and threedimensional systems suggest that this type of symmetry breaking for quantum spin systems only exists in one dimension, the Néel picture always being realized for higher dimensions. By the Néel picture we mean the classical ordering modified by zero-point fluctuations.

\section{Discussion of the results}

In this final section we want to compare the results of the two methods 
available to compute the ground-state energy for the Majumdar-Ghosh chain for values of the parameter $\delta$ in the interval $[-1.00,1.00]$. Both methods make use of a well-defined zero-order ground state, which is modified by fluctuations, which are treated like weakly coupled harmonic oscillators. Both methods are given here in their most simple form, which may be improved by taking into consideration non-harmonic terms in the Hamiltonian. This has been done already for the SWM for the linear chain with nearest-neighbour interactions.

In the method based on cells we neglect all higher-order effects and consequently the effect of the coupling between the cell oscillators on the value of the gap may be underestimated for $\delta \approx \frac{1}{2}$. We want to stress that our method, based on cells, is a very suitable alternative of the standard SWM, which may be clearly illustrated by the results for $\delta \approx 0$ or $\frac{1}{2}$. The SWM gives for $\delta=0$ a value $\bar{\epsilon}_{0}=-3+4 / \pi=-1.7268^{9}$ ) (cf. (28), $\delta=\gamma=\Delta=0$ ) whereas our value for cells of 4 spins equals $\epsilon_{0}^{(4)}=-1.77735$, the exact result being $1-4 \log 2=$ $-1.7726^{16}$ ). For $\delta=\frac{1}{2}$ the SWM does not give a value because this method is restricted to $\delta \leqslant \frac{1}{4}$. Here our method gives, in an almost trivial way, the exact result.

Test for the usefulness of our method for $-1.0 \leqslant \delta \leqslant 1.0$ may be found in the comparison with the exact upper and lower bounds determined by Van den Broek $^{15}$ ). These bounds are listed in table IV under the heading $\epsilon_{U B}$ and $\epsilon_{L B}$. Our values for $\epsilon_{0}^{(4)}$ differ less than $1 \%$ of those for $\epsilon_{U B}$, but are somewhat higher for $0.40 \leqslant \delta \leqslant 0.80$. For other values of the energy per spin we refer to ref. 17 . The spin-wave results, also listed in table IV, are not within Van den Broek's limits, for all possible $\delta \leqslant \frac{1}{4}$ with the exception of $\delta=0.1$.

Summarizing, we may state that our new model for ordered spin chains gives a considerable improvement of the values for the ground state energy as compared to the traditional SWM. We believe that it gives a strong indication of another type of ordering in the interval $0 \leqslant \delta \leqslant 1$.

\section{References}

1) C.K. Majumdar and D.K. Ghosh, J. Math. Phys. 10 (1969) 1399

2) C.K. Majumdar, J. Phys. C3 (1970) 911.

3) P.M. van den Broek, Phys. Lett. 77A (1980) 261.

4) D.J. Klein and T.L. Welsher, J. Stat. Phys. 24 (1981) 555.

5) D.J. Klein, J. Phys. A; Math. Gen. 15 (1982) 661.

6) W.J. Caspers, Physica 115A (1982) 275.

7) W.J. Caspers and W. Magnus, Physica 119A (1983) 291.

8) W.J. Caspers, K.M. Emmitt and W. Magnus, J. Phys. A17 (1984) 2687.

9) P.W. Anderson, Phys. Rev. 86 (1952) 694.

10) P.W. Anderson, Concept of Solids (Benjamin, New York, 1964), p. 157.

11) J. Goldstone, Nuovo Cimento 19 (1961) 154. 
12) W.J. Caspers and W. Magnus, Phys. Lett. 88A (1982) 103.

13) C. Madelung, Introduction to Solid State Theory (Springer, Berlin, 1978), p. 161.

14) N.N. Bogolyubov and S.V. Tyablikov, JETP 19 (1949) 256.

15) P.M. van den Broek, J. Phys. C: Solid State Phys. 13 (1980) 5423.

16) L. Hulthén, Arkiv Math. Astron. Fys. 26A (1983) no. 11.

17) P.M. van den Broek, W.J. Caspers and M.W.M. Willemse, Physica 104A (1980) 298. 